\title{
Behaviors of Microscopic Enterprises and Sectoral Mitigation Policy
}

\author{
Zhugang Jin $^{1,2, a}$, Can Wang ${ }^{1,2, b}$ and Wenjia Cai ${ }^{2, c}$ \\ ${ }^{1}$ School of Environment, Tsinghua University, Beijing China \\ ${ }^{2}$ Center for Earth System Science, Tsinghua University, Beijing China \\ axshs20071727@126.com, ${ }^{\mathrm{b}}$ canwang@tsinghua.edu.cn, cwcai@mail.tsinghua.edu.cn
}

Keywords: sectoral mitigation; microscopic enterprises; net present value; decision model.

\begin{abstract}
From the perspective of microscopic enterprises, a theoretical decision model is put forward and the enterprises' optimal responses to the sectoral mitigation policies are also analyzed. With the simulation of microscopic enterprises' rational behaviors, the "response trap" of policy execution time and the shift of potential switch point due to the policies' influence on marginal profit are evaluated. In order to enhance the efficiency of sectoral mitigation policies, it's optimal to combine both the choice of policy execution time and the policy measures including subsidies, awards, emission taxes and punishments.
\end{abstract}

\section{Introduction}

Sectoral mitigation has been positively promoted by developed countries in global climate negotiation since the Bali Action Plan in 2007. As a new set of options to enhance the effectiveness of greenhouse gas reduction policies, sectoral approach is regarded as a new way to engage the emerging economics on a lower emission path. Baron, et al. [1] introduced the framework design for the sectoral approach, then mitigation potential analyses from sectoral perspectives including electricity, aviation, agriculture and other sectors have been carried out[2-4]. Clapp, et al. [5], Hof and Den Elzen [6] also make comparisons between the national and sectoral approaches. However, apart from few researches focusing on the microscopic enterprise [7], most sectoral mitigation policies are designed upon industrial level, which may weaken the relationship between sectoral policy and the responses of microscopic enterprises. For the rational microscopic enterprises, the execution time of sectoral mitigation policy is not always the right time for the enterprises to carry out reduction and to install clean technology equipments. The subjective initiatives of individual enterprises may enhance or corrode the efficiency of policies. Therefore the effects of the policy execution time, the subsidies, awards and punishments on the initiatives of microscopic enterprises deserve our further investigation, and a theoretical model is also put forward to reveal the relationship in this article.

From the perspective of microscopic enterprises, the sectoral mitigation policy with its simplified impact on the net present value (NPV) of cash flow is introduced in Section 2 covering two scenarios. Then the behaviors of enterprises based on rationality hypothesis are estimated with local and global optimization processes in Section 3. Further discussions and conclusions are made in Section 4 and Section 5 respectively.

\section{NPV and Sectoral Mitigation Policy}

Assume that each microscopic enterprise can maintain a steady average gross profit $\Delta$ in competitive market with the homogenous products. Then without the effects of sectoral mitigation policies, the NPV of cash flow can be calculated as $\int_{0}^{K} \Delta Q \times e^{-\delta s} d s$, where $\mathrm{Q}, \delta$ and $\mathrm{K}$ refer to the quantity of production, discounting rate and life cycle of enterprise respectively. However, the exogenous sectoral mitigation policies together with enterprises' endogenous responses will change the NPVs significantly. In other words, if the execution time of sectoral mitigation policy is fixed to time $\mathrm{H}$, then the rational microscopic enterprises will have incentives to choose the optimal time of mitigation (i.e. t) to minimize the policy shock towards NPV. Generally, compared to policy 
execution time $\mathrm{H}$, the microscopic enterprise's optimal mitigation time $\mathrm{t}$ can be classified into two categories: Active scenario for $\mathrm{t}<\mathrm{H}$, and passive scenario for $\mathrm{t}>\mathrm{H}$.

\subsection{Active Scenario}

For active scenario, the cash flow of the microscopic enterprise can be divided into three stages as Eq. 1 shows:

$$
N P V_{\text {Active }}=\overbrace{\int_{0}^{t} \Delta Q \times e^{-\delta s} d s}^{\text {Stage 1 }}+\overbrace{\int_{t}^{H}(\Delta+l) Q \times e^{-\delta s} d s-A(1-k t) e^{-\delta t}}^{\text {Stage 2 }}+\overbrace{\int_{H}^{K} \Delta Q \times e^{-\delta s} d s}^{\text {Stage 3 }}=\int_{0}^{K} \Delta Q \times e^{-\delta s} d s+\int_{t}^{H} l Q \times e^{-\delta s} d s-A(1-k t) e^{-\delta t} .
$$

During the Stage 1 period, i.e. before the implementation of clean technologies, the enterprise keeps the competitive marginal profit $\Delta$ with the net present value $\int_{0}^{t} \Delta Q \times e^{-\delta s} d s$. During the second period between the implementation $\mathrm{t}$ and the policy execution time $\mathrm{H}$, the marginal profit will be increased to $\Delta+1$ as the results of the subsidy and reward from the government and the excess profit from market with its higher technology, reliability and energy-saving identification together with customer preference. However, the implementation of clean technology requires additional investment which leads to the instantaneous decrease of cash flow. Considering the progress of technology and shift of learning curve, the capital requirement of clean technology implementation is assumed to be $\mathrm{A}(1-\mathrm{kt})$ with the learning curve effect $\mathrm{k}$, where A refers to the initial investment at present. Then after the policy execution time $\mathrm{H}$, the market at Stage 3 will return back to a new steady state with full competition, and the average marginal profit comes back to $\Delta$ again. Hence, the total NPV for active scenario can be reformulated into $\int_{0}^{K} \Delta Q \times e^{-\delta s} d s+\int_{t}^{H} l Q \times e^{-\delta s} d s-A(1-k t) e^{-\delta t}$.

\subsection{Passive Scenario}

Similarly for the passive scenario, if the mitigation time is later than $\mathrm{H}$, then the cash flow of microscopic enterprises can also be divided into three stages:

Before the policy execution time, the competitive marginal profit $\Delta$ is stable with present value $\int_{0}^{H} \Delta Q \times e^{-\delta s} d s$. However, during the period between $\mathrm{H}$ and $\mathrm{t}$, emission charges and regulations together with possible energy-saving identification requirements will reduce the marginal profit to $\Delta-\mathrm{m}$. On the other hand, the enterprise can also take an advantage of the procrastination of clean technology implementation: the technology progress, learning curve shift and discount effect. The latter the technology is implemented, the larger learning and discounting benefit will be enjoyed. After the implementation of clean technology, the negative effect on marginal profit due to sectoral mitigation policy will be released with the marginal profit returning back to $\Delta$. Hence, the total NPV of passive scenario can be calculated as Eq. 2 shows:

$$
N P V_{\text {Passive }}=\int_{0}^{H} \Delta Q \times e^{-\delta s} d s+\int_{H}^{t}(\Delta-m) Q \times e^{-\delta s} d s-A(1-k t) e^{-\delta t}+\int_{t}^{K} \Delta Q \times e^{-\delta s} d s=\int_{0}^{K} \Delta Q \times e^{-\delta s} d s-\int_{H}^{t} m Q \times e^{-\delta s} d s-A(1-k t) e^{-\delta t} .
$$

Generally, Table 1 makes the comparisons of NPV for the active and passive scenarios by segments in detail.

Table 1 Comparison of the NPV for active and passive scenarios by segments

\begin{tabular}{lllll}
\hline Timeline & $0 \sim \min (\mathrm{t}, \mathrm{H})$ & $\min (\mathrm{t}, \mathrm{H}) \sim \mathrm{H}$ & $\mathrm{H} \sim \operatorname{Max}(\mathrm{t}, \mathrm{H})$ & $\operatorname{Max}(\mathrm{t}, \mathrm{H}) \sim \mathrm{K}$ \\
\hline Active & $\int_{0}^{t} \Delta Q \times e^{-\delta s} d s$ & $\left.\int_{t}^{H}(\Delta+l) Q \times e^{-\delta} d s-A(1-l)\right)^{-\delta}$ & 0 & $\int_{H}^{K} \Delta Q \times e^{-\delta s} d s$ \\
Passive & $\int_{0}^{H} \Delta Q \times e^{-\delta s} d s$ & 0 & $\int_{H}^{t}(\Delta-m) Q \times e^{-\delta s} d s-A(1-k t) e^{-\delta \delta}$ & $\int_{t}^{K} \Delta Q \times e^{-\delta s} d s$ \\
\hline
\end{tabular}

\section{Behaviors of Enterprises}

Given the exogenous policy execution time $\mathrm{H}$, the rational microscopic enterprises will not only determine the active or passive strategy, but also the implementation time t. Firstly, the local optimal implementation time for each strategy should be worked out together with the maximal NPVs respectively. Secondly, the global optimal implementation time is selected by comparing the NPVs. Generally, the global optimal implementation time $t$ meet the objective of maximizing the $\mathrm{NPV}_{\text {Global }}$ as Eq. 3 shows where $\Phi(x)$ represents a 0 -1 function. If $x \geq 0, \Phi(x)=1$, and if $x<0$, then $\Phi(x)=0$. In order to simplify the model, we assume that $\mathrm{Q}$, the quantity of product is consistent. 


$$
N P V_{\text {Global }}=\int_{0}^{K} \Delta Q \times e^{-\delta s} d s+\Phi(H-t) \times \int_{t}^{H} l Q \times e^{-\delta s} d s-\Phi(t-H) \int_{H}^{t} m Q \times e^{-\delta s} d s-A(1-k t) e^{-\delta t}
$$

\subsection{Local Optimization}

Leaving the restriction $\mathrm{t}<\mathrm{H}$, the local optimal time for active scenario can be further worked out by maximizing $\mathrm{NPV}_{\text {Active }}$ with the local optimal time $\mathrm{T}_{\mathrm{a}}$ as Eq. 4 shows, and the local maximized NPV equals to $\mathrm{NPV}_{\text {Active }}=\mathrm{T}$ a . However, if $\mathrm{T}_{\mathrm{a}}$ is larger than $\mathrm{H}$, then the adjusted local optimal time will be restricted to $H$ because $T_{a}$ under this condition is infeasible.

$$
T_{a}=\frac{A k+A \delta-l Q}{A k \delta}=\frac{1}{\delta}+\frac{1}{k}-\frac{l Q}{A k \delta} .
$$

Similarly, the local optimal time for passive scenario can also be worked out by maximizing $N P V_{\text {Passive. }}$. The unrestricted local optimal time $T_{b}$ can be expressed as Eq. 5 . However, if $T_{b}$ is smaller than $\mathrm{H}$, then the adjusted local optimal time will be restricted to $\mathrm{H}$ because $\mathrm{T}_{\mathrm{b}}$ under $\mathrm{t}<\mathrm{H}$ restriction is also infeasible.

\subsection{Global Optimization}

$$
T_{b}=\frac{A k+A \delta-m Q}{A k \delta}=\frac{1}{\delta}+\frac{1}{k}-\frac{m Q}{A k \delta} .
$$

Compared to the endogenous local optimal time preference $T_{a}$ and $T_{b}$, the exogenous policy information $\mathrm{H}$ is more flexible, which means $\mathrm{H}$ is not always larger than $\mathrm{T}_{\mathrm{a}}$ or $\mathrm{T}_{\mathrm{b}}$. In addition, the extra increment 1 and decrement $\mathrm{m}$ on marginal profit is also highly dependent on the strictness of sectoral mitigation policies, then the relationship of size between $T_{a}$ and $T_{b}$ is not stable, not to mention $\mathrm{H}$. Therefore, with the local optimal time information, the global optimization can be further discussed with following six cases as Table 2 shows. Table 3 illustrates the basic illustrations of the

\begin{tabular}{|c|c|c|c|c|c|c|}
\hline \multirow[b]{2}{*}{ Cases } & \multicolumn{3}{|c|}{$1 \geq \mathrm{m}$} & \multicolumn{3}{|c|}{$1<\mathrm{m}$} \\
\hline & $\begin{array}{l}H<T_{a}<T_{b} \\
(1-1)\end{array}$ & $\begin{array}{l}T_{a}<H<T_{b} \\
(1-2)\end{array}$ & $\begin{array}{l}T_{a}<T_{b}<H \\
(1-3)\end{array}$ & $\begin{array}{l}H<T_{b}<T_{a} \\
(2-1)\end{array}$ & $\begin{array}{l}T_{b}<H<T_{a} \\
(2-2)\end{array}$ & $\begin{array}{l}T_{b}<T_{a}<H \\
(2-3)\end{array}$ \\
\hline $\begin{array}{l}\text { Global } \\
\text { Optimal } \\
\text { time }\end{array}$ & $\mathrm{T}_{\mathrm{b}}$ & $\mathrm{T}_{\mathrm{a}}$ or $\mathrm{T}_{\mathrm{b}}$ & $\mathrm{T}_{\mathrm{a}}$ & $\mathrm{T}_{\mathrm{b}}$ & $\mathrm{H}$ & $\mathrm{T}_{\mathrm{a}}$ \\
\hline $\begin{array}{l}\text { maximized } \\
\mathrm{NPV}_{\text {Global }}\end{array}$ & $\mathrm{NPV}_{\text {Passivel}}=\mathrm{Tb}$ & $\begin{array}{l}\mathrm{NPV}_{\text {Activel } \mid=T a} \\
\text { or } \\
\mathrm{NPV} \\
\text { Passivel }=\mathrm{Tb}\end{array}$ & $\mathrm{NPV}_{\text {Active } \mid=T a}$ & $N P V_{\text {Passivelt }=\mathrm{Tb}}$ & $\mathrm{NPV}_{\mathrm{H}}$ & $\mathrm{NPV}_{\text {Activel } \mid=T a}$ \\
\hline
\end{tabular}
global optimization processes respectively.

Table 2 Theoretical results of global optimal time (six cases)

\section{Discussion}

The global optimization results offer us a new perspective to investigate the influences of sectoral mitigation policy on microscopic enterprises' behaviors together with the enterprises' internal strategies.

Firstly, cases (1-1) and (2-1) indicate that if the policy execution time $\mathrm{H}$ is much more urgent, the outcome of microscopic enterprises' behaviors will be independent and consistent by choosing the passive strategy. Similarly, cases (1-3) and (2-3) also show that if $\mathrm{H}$ is very loose, the global optimal behaviors of rational enterprises will be consistent by choosing the active strategy. In other words, if the policy execution time $\mathrm{H}$ is smaller or larger than the lower and upper boundaries (i.e. $\min \left(\mathrm{T}_{\mathrm{a}}, \mathrm{T}_{\mathrm{b}}\right.$ ) and $\max \left(\mathrm{T}_{\mathrm{a}}, \mathrm{T}_{\mathrm{b}}\right)$ respectively) without additional policy influence on the marginal profit (i.e. 1 and $\mathrm{m}$ ), the behaviors of rational enterprises will be stable without any response to the change of $\mathrm{H}$. To some extent, there is a "response trap" for policy execution time $\mathrm{H}$.

Secondly, there is a potential switch point $\mathrm{T}_{\text {switch }}$ for the microscopic enterprises as Eq. 6 shows. When the marginal increment effect of sectoral mitigation utility on profit (i.e. l) is larger than the decrement effect $m$, then the switch point for the enterprises is an interval $\left[T_{b}, T_{a}\right]$. It means that if the policy execution time $\mathrm{H}$ is smaller than $\mathrm{T}_{\text {switch }}$, then the consistent response of rational microscopic enterprises is the passive strategy, i.e. the global optimal time of clean technology implementation is latter than $\mathrm{H}$. On the country, if the policy execution time $\mathrm{H}$ is larger than the potential switch point, 
then the consistent response of rational enterprises is active strategy, i.e. the global optimal time of clean technology implementation will be ahead of $\mathrm{H}$. Similarly, when $\mathrm{l} \geq \mathrm{m}$, the potential switch point $\mathrm{T}_{\text {switch }}$ can also be worked out. In addition, for case (1-2) when $\mathrm{T}_{\mathrm{a}}<\mathrm{H}<\mathrm{T}_{\mathrm{b}}$, the optimal implementation time depends on the comparison of $\mathrm{NPV}_{\text {Active }} \mathrm{t}=\mathrm{Ta}$ and $\mathrm{NPV}_{\text {Passive }} \mathrm{t}=\mathrm{Tb}$. Only if $\mathrm{t}>\frac{1}{\delta}+\frac{1}{k}-\frac{1}{\delta}\left[\ln \left(e^{\frac{I Q}{A n}}-e^{\frac{m Q}{A n}}\right)-\ln \left(\frac{l Q}{A n}-\frac{m Q}{A n}\right)\right]$, the rational strategy is active, which means that the $\mathrm{T}_{\text {switch }}$ under condition of $1 \geq \mathrm{m}$ refers to a point rather than an interval. Furthermore, the switch point $\mathrm{T}_{\text {switch }}$ gives us a reference standard to evaluate the effectiveness of sectoral mitigation policy. If the execution time of sectoral mitigation policy is too urgent, it can hardly trigger the early initiative responses.

$$
T_{\text {swich }}=\left\{\begin{array}{l}
{\left[\frac{1}{\delta}+\frac{1}{k}-\frac{m Q}{A n \delta}, \frac{1}{\delta}+\frac{1}{k}-\frac{l Q}{A n \delta}\right] \text { if } l<m} \\
\frac{1}{\delta}+\frac{1}{k}-\frac{1}{\delta}\left[\ln \left(e^{\frac{l Q}{A n}}-e^{\frac{m Q}{A n}}\right)-\ln \left(\frac{l Q}{A n}-\frac{m Q}{A n}\right)\right] \text { if } l \geq m
\end{array} .\right.
$$

Thirdly, apart from the "response trap" as the result of purely time $\mathrm{H}$, the additional increment and decrement of marginal profit highly relevant to the sectoral mitigation policies can affect the switch point significantly as Fig.1 shows. With Eq. 6, we can further work out the marginal relation that $\frac{\partial T_{\text {switch }}}{\partial l}<0$ and $\frac{\partial T_{\text {switch }}}{\partial m}<0$, which means the larger 1 or $\mathrm{m}$, the smaller the switch point will be. It means that if policy makers offer more attractive rewards and subsidies to enhance the marginal increment 1 for the active enterprises, the switch point will be smaller and microscopic enterprises will have more incentives to choose the active strategy under the same policy execution time H. Similarly, if the policy makers carry out more strict regulations and severe punishment for excess emissions which leads to the higher $m$, the rational enterprises will degrade the switch point consciously and choose the active strategy with greater probability and incentives.

Generally, the adjustment of policy execution time $H$ and marginal profit in/decrement $1 / \mathrm{m}$ influence the behaviors of microscopic enterprises from two different perspectives: the adjustment of $\mathrm{H}$ can hardly affect the endogenous incentives of enterprises as the rational switch point is independent on $\mathrm{H}$, however, the adjustment of 1 and $\mathrm{m}$ can affect the endogenous switch points directly, which is much more significant and efficient than $\mathrm{H}$ to some extent. The different probabilities of policy execution time $\mathrm{H}$ and marginal profit influence $1 / \mathrm{m}$ indicate that it's a good choice to combine both methods to enhance the efficiency of sectoral mitigation policies.

Table 3 Illustrations of six cases for global optimization processes

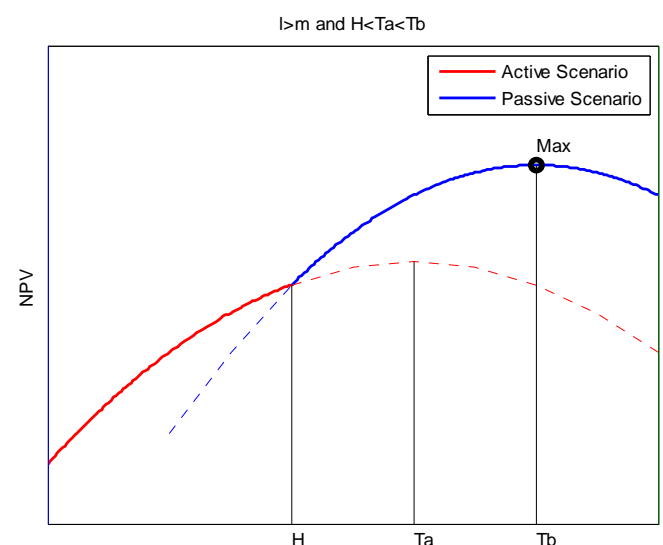

$(1-1)$

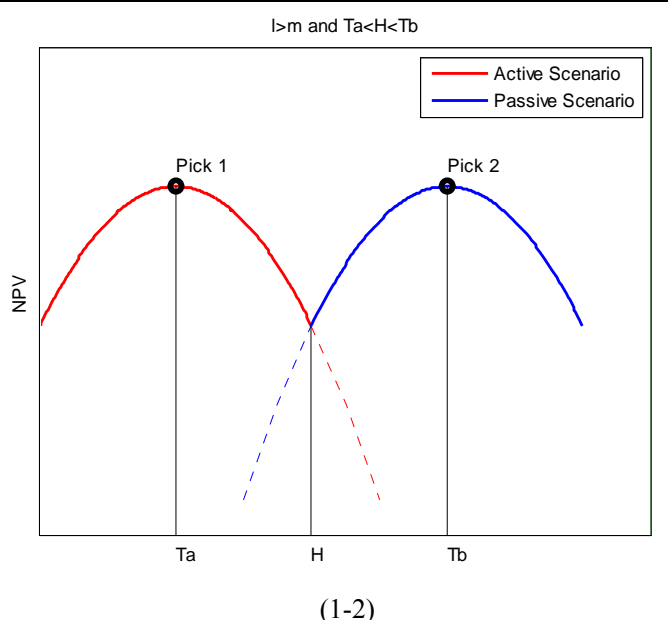

$(1-2)$ 


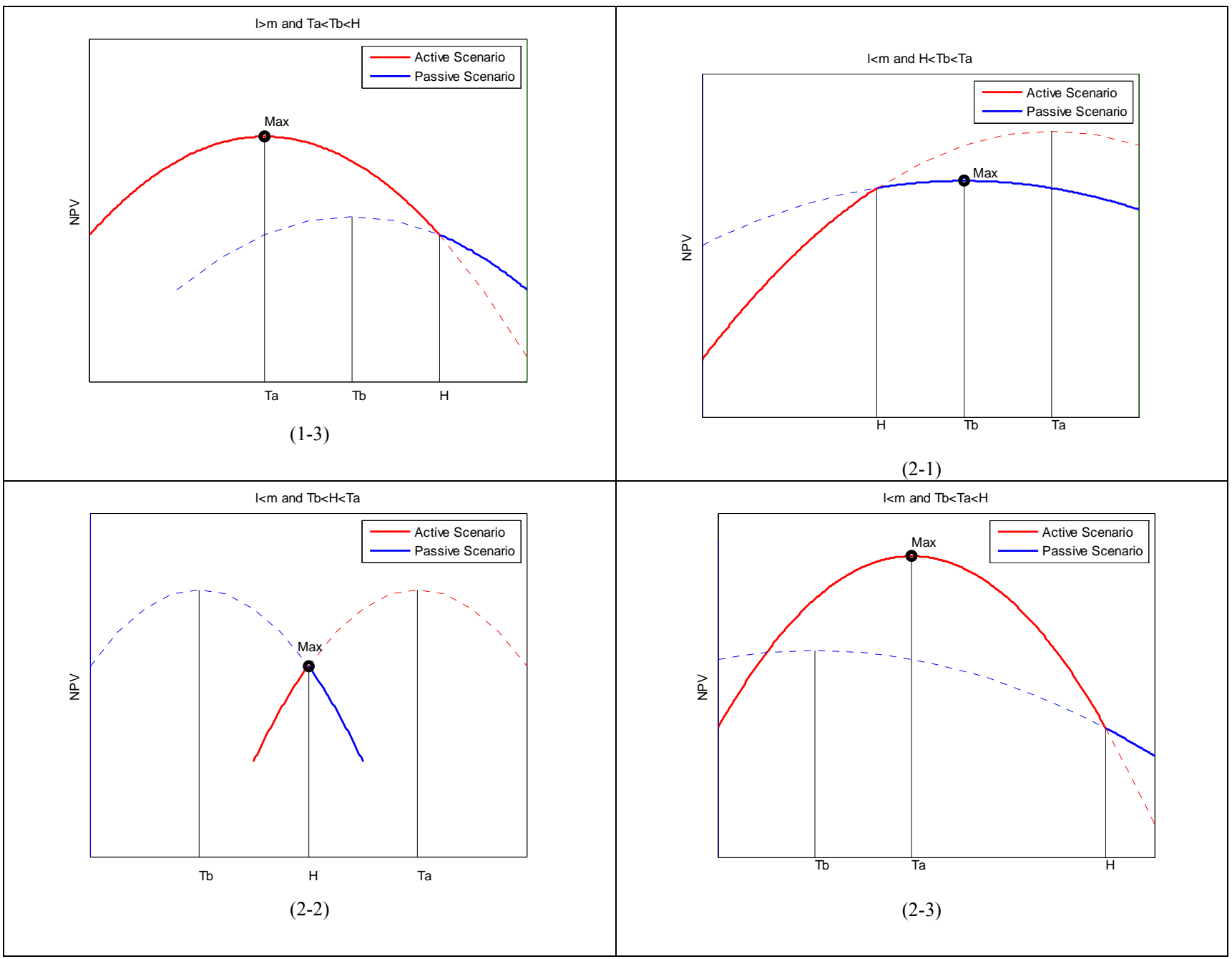

Notes: dashed lines refer to the infeasible situations and real lines for the feasible ones. Blue lines stand for Passive scenarios and read lines for Active scenarios.

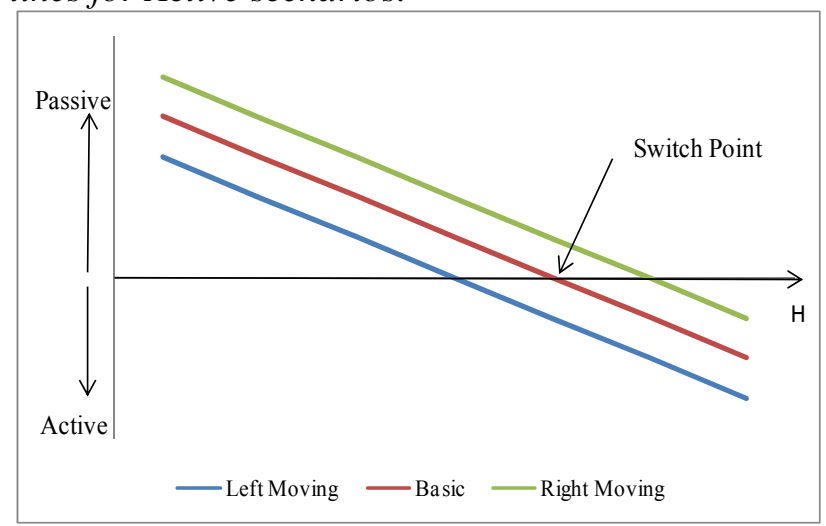

Fig. 1: The NPV difference, switch point and strategy selections

\section{Conclusions}

By taking the subjective initiatives of microscopic enterprises into consideration, the efficiency of sectoral mitigation policies is reevaluated with a theoretical decision model. Theoretical results indicate that there is a "response trap" for the execution time of sectoral mitigation policies, which means if the policy execution time is out of a specific range, the change of policy execution time will have no influence on the microscopic enterprises' decisions and behaviors. However, the policy measures including subsidies, awards for reduction in advance and taxes, punishments for excess emissions after the execution of policy will significantly induce the shift of microscopic enterprises' potential switch point. With more attractive subsidies, awards and more severe taxes, punishment, the 
microscopic enterprises have more incentives to carry out the mitigation of greenhouse gases in spite of the execution time of sectoral mitigation policies. Therefore, in order to maximize the efficiency of sectoral mitigation polices, it's optimal to combine both the choice of execution time and specific measures including subsidies, awards, taxes and punishments.

\section{Acknowledgement}

This work was financially supported by the National Natural Science Foundation of China (No.71273153).

\section{References}

[1] R. Baron, J. Reinaud, M. Genasci, C. Philibert, "Sectoral Approaches to Greenhouse Gas Mitigation-Exploring Issues for Heavy Industry," IEA information paper, 2007.

[2] W. Cai, C. Wang, J. Chen, "Revisiting $\mathrm{CO}_{2}$ mitigation potential and costs in China's electricity sector," Energy Policy. 2010, 38: 4209-4213.

[3] K. Hihara, "Analysis on bargaining about global climate change mitigation in international aviation sector," Transportation Research Part E: Logistics and Transportation Review. 2011, 47: 342-358.

[4] J. Wang, J. Huang, S. Rozelle, "Climate Change and China's Agricultural Sector: An Overview of Impacts," Adaptation and Mitigation, 2010.

[5] C. Clapp, K. Karousakis, B. Buchner, J. Chateau, "National and sectoral GHG mitigation potential: A comparison across models," Organisation for Economic Co-operation and Development (OECD). 2009.

[6] A.F. Hof, M.G. Den Elzen, "The effect of different historical emissions datasets on emission targets of the sectoral mitigation approach Triptych," Climate Policy. 2010, 10: 684-704.

[7] H. Zeppel, N. Beaumont, "Assessing motivations for carbon offsetting by environmentally certified tourism enterprises," Anatolia. 2013: 1-22. 